\title{
Opiniões e atitudes dos consumidores em relação a embalagens e rótulos de cachaça
}

\author{
Consumers'opinion and views on packaging and labels of cachaça
}

\author{
João de Deus Souza CARNEIROํ, Valéria Paula Rodrigues MINIM², \\ Jose Benicio Paes CHAVES ${ }^{2}$, Carlos Henrique Osório SILVA³ ${ }^{3}$, Adair José REGAZZI ${ }^{3}$
}

\section{Resumo}

A técnica de grupo de foco foi utilizada para obter informações detalhadas a respeito das opiniões e atitudes dos consumidores em relação a embalagens e rótulos de cachaça. Objetivou-se gerar informações para os produtores do setor de cachaça e aumentar a competitividade desse produto no mercado. Foram realizadas três sessões de 90 minutos com oito participantes em cada. Quatro embalagens de cachaça foram apresentadas, separadamente e, para cada apresentação, foi seguido um roteiro de perguntas, previamente elaborado. Verificou-se que os participantes não aprovam a garrafa de vidro de cor âmbar com tampa metálica tipo coroa (igual à da garrafa de cerveja) para acondicionar cachaça, porém preferem a garrafa de vidro incolor com capacidade igual a $700 \mathrm{~mL}$ e com tampa metálica rosqueável e ilustrações do rótulo que estejam relacionadas com o processo produtivo de cachaça. A marca, selos de qualidade, premiações recebidas e as informações a respeito do local onde a cachaça foi produzida, do tempo de envelhecimento e do tipo de madeira do tonel no qual a cachaça foi envelhecida também influenciam na compra de cachaça. Observou-se ainda que os participantes apresentaram um padrão de preferência, sendo a maioria influenciada pelos mesmos fatores, no momento da compra de cachaça.

Palavras-chave: grupo de foco; cachaça; comportamento; consumidor; competitividade; embalagem.

\begin{abstract}
The focus group technique was used to obtain detailed information about the consumers' opinion and views on packaging and labels of cachaça to generate information for cachaça producers and increase the competitiveness of this product on the market. Three 90 minutepanel sessions with eight participants each were held. Four different packages of cachaça were presented separately, and each presentation was followed by a set of questions previously elaborated. It was found that the panelists disliked the amber glass bottle with metal crown caps (similar to the beer's bottle cap). Nevertheless, it was noted a preference for the transparent glass bottle, $700 \mathrm{~mL}$ capacity, with screw top and label illustrations related to the cachaça's productive process. Details such as brand, quality seals, awards received and information about the place where the cachaça was produced, ageing, and the type of wood of the barrel in which the cachaça was aged influence the purchase of cachaça. It was also observed that the panelists presented a preference standard; the majority was influenced by the same factors at the time of purchase of cachaça.

Keywords: focus group; cachaça; behavior; consumer; competitiveness; packaging.
\end{abstract}

\section{Introdução}

A cachaça representa importante papel para a economia do país, sendo responsável pela geração de emprego e renda, principalmente, para pequenos produtores rurais. Atualmente, existem 30 mil produtores no Brasil e a produção de cachaça é de 1,3 bilhão de L anuais (ASSOCIAÇÃO BRASILEIRA..., 2009). Ressalta-se que a produção de cachaça é uma atividade em expansão no País, sendo a cachaça a segunda bebida alcoólica mais consumida, ficando atrás apenas da cerveja, e com perspectivas de expansão no mercado externo.

A cachaça durante várias décadas foi considerada como uma bebida das classes mais pobres, vendida a preços baixos e produzida sem controle de qualidade. Nas últimas décadas, foram realizadas várias ações e programas para o desenvolvimento do agronegócio da cachaça, tais como: modificação da legislação, criação de associações de produtores de cachaça de qualidade e investimentos em controle de qualidade, pesquisa e marketing.

Estas ações e programas objetivaram valorizar a cachaça, aumentar a aceitação do produto no mercado interno e externo, mudar seu status e transformar uma atividade historicamente informal em outra, voltada para o mercado e para a competitividade (FEDERAÇÃO..., 2007; ASSOCIAÇÃO MINEIRA..., 2009; SERVIÇO..., 2001).

Entretanto, a literatura brasileira é escassa de trabalhos a respeito do comportamento dos consumidores de cachaça. A compreensão do comportamento, ou seja, entender como as pessoas compram e usam os produtos, auxilia as empresas a satisfazerem as necessidades dos consumidores e contribui para o sucesso do produto no mercado.

${ }^{1}$ Ciência dos Alimentos, Universidade Federal de Lavras - UFL, CEP 37200-000, Lavras - MG, Brasil

2 Departamento de Tecnologia de Alimentos, Universidade Federal de Viçosa - UFV, CEP 36570000, Viçosa - MG, Brasil, E-mail: vprm@ufv.br

${ }^{3}$ Departamento de Informática e Estatística, Universidade Federal de Viçosa - UFV, CEP 36570000, Viçosa - MG, Brasil

${ }^{*}$ A quem a correspondência deve ser enviada 
Entre os fatores que influenciam o comportamento dos consumidores, destacam-se os fatores da embalagem e do rótulo dos produtos. A embalagem e o rótulo possuem fundamental importância na escolha do produto durante a compra, uma vez que representam o primeiro contato entre o consumidor e o produto. Assim, para se otimizar o processo de escolha, compra e aceitação da cachaça, é interessante que se identifiquem os fatores da embalagem e do rótulo valorizados pelos consumidores.

Um dos métodos de pesquisa, que pode ser utilizado para se obter as opiniões e atitudes dos consumidores em relação às embalagens e rótulos de produtos, é a técnica de grupo de foco. O grupo de foco é uma das mais populares formas de pesquisa com consumidores e é definido como uma entrevista cuidadosamente planejada, com o objetivo de obter as atitudes e as opiniões das pessoas a respeito de determinados assuntos, produtos ou serviços (DELLA LUCIA; MINIM, 2006).

Dentro deste contexto, este trabalho utilizou a técnica de grupo de foco para obter informações detalhadas a respeito das opiniões e atitudes dos consumidores em relação às embalagens e rótulos de cachaça, objetivando gerar informações para os produtores do setor e aumentar a competitividade desse produto no mercado.

\section{Material e métodos}

Os grupos de foco foram planejados e realizados de acordo com as etapas descritas por Della Lucia e Minim (2006).

Foram distribuídos 100 questionários de recrutamento entre pessoas residentes na cidade de Viçosa - MG. Estes questionários foram distribuídos em estabelecimentos que comercializavam cachaça e na Universidade Federal de Viçosa. De acordo com as respostas obtidas, por meio dos questionários, foram selecionadas 24 pessoas, as quais consumiam cachaça pelo menos uma vez por mês e tinham o hábito de ler o rótulo das cachaças no momento da compra e de frequentar supermercados para realizar compras.
Três sessões de grupos de foco foram conduzidas por um único moderador em uma sala com capacidade para acomodar os participantes confortavelmente. Estes se sentaram em torno de uma mesa redonda para permitir a interação, o contato visual e a harmonia da discussão. Cada sessão durou, aproximadamente, 90 minutos e teve a participação de oito pessoas.

No início de cada sessão, o moderador apresentou o propósito da técnica, o papel do moderador, o objetivo do estudo, e cada participante se apresentou ao grupo. Aos participantes foi assegurado que não existiam respostas certas ou erradas para as questões abordadas e foram encorajados a expressar suas opiniões, mesmo que estas fossem divergentes das respostas dos demais membros do grupo.

Iniciou-se a discussão com perguntas a respeito da opinião dos consumidores e suas atitudes em relação às embalagens e rótulos de cachaça. Em seguida, embalagens rotuladas e contendo cachaça de quatro marcas comerciais foram apresentadas (Quadro 1). Estas foram apresentadas separadamente e cada apresentação foi seguida por um roteiro de perguntas previamente elaborado (Quadro 2). Para cada sessão utilizou-se uma ordem diferente de apresentação das embalagens, sendo esta aleatória. As cachaças utilizadas neste estudo foram adquiridas no comércio de Viçosa - MG.

As sessões de grupos de foco foram anotadas e gravadas por um assistente. A partir das anotações e gravações, obtiveram-se as respostas dos 24 participantes, a respeito das embalagens e rótulos de cachaça.

Os dados obtidos por meio do questionário de recrutamento e das sessões de grupos de foco foram submetidos à análise de frequência, utilizando-se o programa SAS, versão 9.0, licenciado para a Universidade Federal de Viçosa.

\section{Resultados e discussão}

O resumo das características demográficas dos participantes dos grupos de foco está apresentado na Tabela 1. A maioria deles era do sexo masculino (95,8\%) e 70,8\% tinham idade entre

Quadro 1. Descrição das embalagens e rótulos das cachaças apresentadas nas sessões de grupo de foco.

\begin{tabular}{|c|c|c|}
\hline Identificação/marca & Tipo de embalagem & Descrição do rótulo \\
\hline $\begin{array}{l}1 \text { - Cachaça da } \\
\text { marca A }\end{array}$ & $\begin{array}{l}\text { Garrafa de vidro transparente com capacidade } \\
\text { igual a } 700 \mathrm{~mL} \text { e tampa metálica rosqueável. }\end{array}$ & $\begin{array}{l}\text { Rótulo preto com ilustração (desenho) de uma árvore e com selo da AMPAQ. } \\
\text { No painel frontal constavam as seguintes informações escritas na cor verde: } \\
\text { nome da marca, relação dos prêmios recebidos em festivais de cachaça, } \\
\text { conteúdo líquido de } 700 \mathrm{~mL} \text { e cachaça de Minas. }\end{array}$ \\
\hline $\begin{array}{l}2 \text { - Cachaça da } \\
\text { marca B }\end{array}$ & $\begin{array}{l}\text { Garrafa de vidro transparente com capacidade } \\
\text { igual a } 1000 \mathrm{~mL} \text { e tampa plástica rosqueável. }\end{array}$ & $\begin{array}{l}\text { Rótulo verde e dourado com ilustração (foto) de alambiques de cobre e com } \\
\text { selo da AMPAQ. No painel frontal constavam as seguintes informações } \\
\text { escritas na cor dourada: nome da marca, envelhecida em tonéis de carvalho } \\
3 \text { anos, cachaça de Minas e conteúdo líquido de } 1000 \mathrm{~mL} \text {. }\end{array}$ \\
\hline $\begin{array}{l}3 \text { - Cachaça da } \\
\text { marca C }\end{array}$ & $\begin{array}{l}\text { Garrafa de vidro transparente com capacidade } \\
\text { igual a } 700 \mathrm{~mL} \text { e tampa metálica rosqueável. }\end{array}$ & $\begin{array}{l}\text { Rótulo marrom com ilustração (desenho) de trabalhadores colhendo cana- } \\
\text { de-açúcar. No painel frontal constavam as seguintes informações escritas na } \\
\text { cor marrom: nome da marca, armazenada em tonéis de umburana, cachaça de } \\
\text { Minas e conteúdo líquido de } 700 \mathrm{~mL} \text {. }\end{array}$ \\
\hline $\begin{array}{l}4 \text { - Cachaça da } \\
\text { marca D }\end{array}$ & $\begin{array}{l}\text { Garrafa de vidro âmbar com capacidade } \\
\text { igual a } 600 \mathrm{~mL} \text { e tampa metálica tipo } \\
\text { coroa (semelhante a da garrafa utilizada no } \\
\text { acondicionamento de cerveja). }\end{array}$ & $\begin{array}{l}\text { Rótulo amarelo sem ilustração. No painel frontal constavam as seguintes } \\
\text { informações escritas na cor vermelha: nome da marca e conteúdo líquido de } \\
600 \mathrm{~mL} \text {. }\end{array}$ \\
\hline
\end{tabular}


20 e 49 anos. Em relação ao grau de instrução: $29,2 \%$ tinham pós-graduação; $20,8 \%$ tinham curso superior completo; $25,0 \%$ tinham curso superior incompleto; e $25,0 \%$, apenas $2^{\circ}$ grau. Quanto à renda familiar: 29,2\% dos participantes tinham renda entre 1 e 5 salários mínimos; 45,8\%, entre 5 e 10 salários mínimos; e 25,0\%, entre 10 e 20 salários mínimos.

O perfil dos participantes dos grupos de foco foi diferente do perfil da população brasileira em relação às variáveis faixa etária, sexo, renda e grau de instrução. Este resultado sugere que o mercado consumidor de cachaça é constituído, em sua maioria, por pessoas do sexo masculino e com maior grau de instrução e renda. Isto reforça a constatação de Aguiar (2004), segundo a qual a cachaça deixou de ser, somente, bebida de botequins e das classes mais pobres, passando a ser consumida em bares e ambientes mais sofisticados. Esse autor ressalta ainda que o mercado de cachaça no Brasil tem passado por recentes transformações, mostradas, principalmente, por certa elitização do produto.

Em relação à frequência de consumo, todos os participantes dos grupos de foco consumiam cachaça pelo menos uma vez por mês, sendo que $8,0 \%$ consumiam diariamente; $42,0 \%$, uma vez por semana; $21,0 \%$, duas vezes por mês; e 29,0\%, uma vez por mês (Figura 1).

A frequência de leitura dos rótulos de produtos pelos participantes dos grupos de foco está apresentada na Figura 2. Verificou-se que $62,5 \%$ sempre leem os rótulos dos produtos; $25,0 \%$ leem às vezes; e $12,5 \%$ leem frequentemente.

De acordo com os resultados das sessões de grupo de foco, preço, marca, ilustração e cor do rótulo e informações sobre o conteúdo líquido, graduação alcoólica e lote são alguns dos fatores do rótulo que os participantes dos grupos de foco observam no momento da compra de cachaça. A marca é observada por todos os participantes; o preço por $83 \%$; a ilustração do rótulo por $79 \%$; a cor do rótulo por $75 \%$; a informação sobre o conteúdo líquido por 67\%; a informação sobre graduação alcoólica por $61,5 \%$; e a informação sobre o lote por $17 \%$.

Os participantes disseram que a marca é um fator muito importante no processo de escolha e compra de cachaça e que preferem comprar marcas conhecidas. Esse resultado coincide

Quadro 2. Roteiro de perguntas para as sessões de grupo de foco.

Você observa os rótulos das cachaças que consome?
O que você observa?
O que mais chama a sua atenção?
O que você achou desta embalagem?
O que você considera importante nesta embalagem?
Você gostaria de ver alguma outra informação no rótulo?
Você observa o tempo de envelhecimento e o tipo de madeira do tonel,
no qual a cachaça foi envelhecida, no momento da compra?
Qual é sua opinião em relação aos selos de qualidade e prêmios
recebidos pela cachaça?
Você observa o local onde a cachaça foi produzida no momento da
compra?

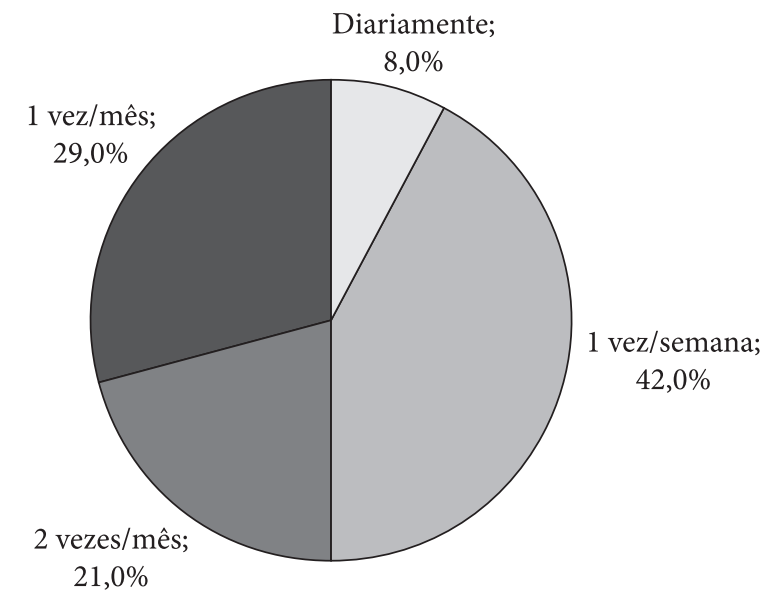

Figura 1. Frequência de consumo de cachaça dos participantes dos grupos de foco.

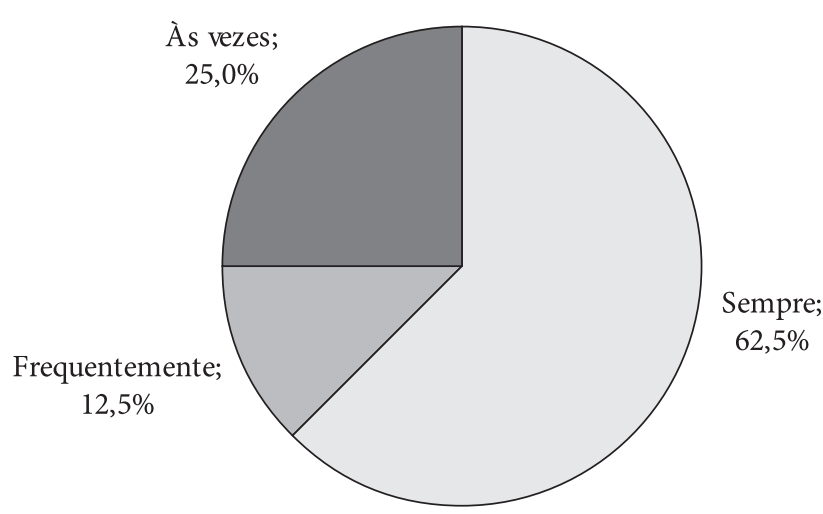

Figura 2. Frequência de leitura dos rótulos de produtos pelos participantes dos grupos de foco.

Tabela 1. Resumo das características demográficas dos participantes dos grupos de foco $(\mathrm{n}=24)$.

\begin{tabular}{lcc}
\hline \multicolumn{1}{c}{ Variáveis demográficas } & Classes & $\%$ \\
\hline Sexo & Feminino & 4,2 \\
Faixa etária (anos) & Masculino & 95,8 \\
& $20-29$ & 45,8 \\
& $30-39$ & 12,5 \\
& $40-49$ & 12,5 \\
& $50-59$ & 20,8 \\
& $60-69$ & 4,2 \\
& Acima de 70 & 4,2 \\
$1^{\circ}$ grau & 0,0 \\
$2^{\circ}$ grau & 25,0 \\
Renda familiar mensal & Superior incompleto & 25,0 \\
(salários mínimos) & Superior completo & 20,8 \\
& Pós-graduação & 29,2 \\
& 1 a 5 & 29,2 \\
\hline
\end{tabular}


com os obtidos por Reis (2007), Carneiro (2002), Costa (1999) e Deliza (1996). Nos trabalhos realizados por estes pesquisadores, os produtos de marcas conhecidas foram preferidos pelos consumidores, os quais consideraram estes como de melhor qualidade que aqueles de marcas desconhecidas. Assim, é fundamental que os produtores de cachaça elaborem estratégias de marketing, visando tornar suas marcas conhecidas.

Observou-se que $79 \%$ dos participantes gostam de ilustrações que estejam relacionadas com o processo produtivo de cachaça, como plantação de cana-de-açúcar, carro de boi, tonéis, alambiques, etc. Em relação às ilustrações dos rótulos das cachaças apresentadas, os participantes não apreciaram a ilustração do rótulo da cachaça da marca $\mathrm{A}$ e disseram que o rótulo da cachaça da marca $\mathrm{D}$ era muito simples, pois não apresentava ilustração. Nos trabalhos realizados por Costa (1999) e Deliza (1996), verificou-se, também, que a ilustração do rótulo foi um fator que representou um importante papel na preferência e na intenção de compra dos consumidores de óleo de girassol e suco de maracujá, respectivamente.

Em relação à cor do rótulo, detectou-se que os participantes gostaram das cores dos rótulos das cachaças das marcas B e C. Disseram que a cor vermelha, presente no rótulo da cachaça da marca $\mathrm{D}$, não é adequada para rótulo de cachaça. Quanto ao rótulo da cachaça da marca A, 29\% dos participantes não apreciaram a cor preta. Nas sessões de grupo de foco realizadas por Della Lucia (2005) e Dantas (2001), a cor do rótulo também foi identificada como um fator que influência na intenção de compra dos consumidores de café torrado e moído e de couve minimamente processada, respectivamente.

Outro fator que influência no processo de compra de cachaça é o tipo e fechamento da garrafa. Verificou-se que $100 \%$ dos participantes não aprovam a garrafa de vidro de cor âmbar para acondicionar cachaça. Observou-se também que $75 \%$ dos participantes preferem a garrafa de vidro incolor transparente, com capacidade igual a $700 \mathrm{~mL} ; 21 \%$ preferem a de vidro incolor, com capacidade igual a $1000 \mathrm{~mL}$; e $4 \%$ gostam de ambas as garrafas. Os participantes disseram que a garrafa de vidro de cor âmbar não agrega valor à cachaça, não permite sua visualização e é relacionada com garrafa reciclada. A garrafa de vidro transparente com capacidade igual a $700 \mathrm{~mL}$ é atraente, prática, permite a visualização da cachaça e lhe agrega valor.

Em relação ao fechamento da garrafa, todos os participantes não gostaram da tampa metálica tipo coroa (igual à da garrafa de cerveja); $75 \%$ preferem a tampa metálica rosqueável; $21 \%$, a plástica rosqueável; e $4 \%$, a rolha de cortiça. Os participantes disseram que não é possível reutilizar a tampa metálica tipo coroa para fechar adequadamente a garrafa de cachaça, após esta ser aberta. Os mesmos ressaltaram, ainda, que este fato causa grande inconveniente, pois não se consome uma garrafa de cachaça de uma única vez.

Os consumidores que preferem a tampa plástica rosqueável e a rolha de cortiça alegaram que elas proporcionam um fechamento hermético da garrafa, tanto antes quanto depois de ser aberta.

Notou-se também que 29,0\% dos participantes não aprovam a tampa metálica rosqueável, pois, segundo eles, algumas dessas tampas garantem o fechamento hermético da garrafa antes de ser aberta pela primeira vez, inviabilizando, assim, a reutilização deste tipo de tampa para esta finalidade.

Em trabalhos que avaliaram a influência dos fatores da embalagem na preferência dos consumidores de óleo de soja (CARNEIRO, 2002), mel (MURPHY; COWAN; HENCHION, 2000) e presunto (STEENKAMP, 1987), o fator tipo de embalagem também influenciou no processo de escolha, compra e aceitação dos consumidores.

Quanto às informações a respeito do tempo de envelhecimento e do tipo de madeira do tonel, no qual a cachaça foi envelhecida, todos os participantes consideram estas informações importantes e as observam no momento da compra da bebida. Verificou-se que há preferência, entre os participantes, por cachaça envelhecida em tonéis de carvalho, umburana e jequitibá, respectivamente.

Detectou-se ainda que todos os participantes observam o local ou região onde a cachaça foi produzida no momento da compra, tendo 75\% dito que a informação "Cachaça de Minas" é um diferencial e influência positivamente na compra de cachaça. Esse resultado é semelhante ao obtido por Bernabéu e Tendero (2005) e por Moskowitz et al. (2004), os quais identificaram que o fator origem influenciou na preferência dos consumidores de carne de cordeiro e azeitona, respectivamente.

Duas embalagens tinham o selo da AMPAQ, logo, perguntou-se aos participantes suas opiniões em relação aos selos de qualidade. A maioria dos participantes (75\%) considera os selos de qualidade importantes no momento da compra, $17 \%$ não confiam neles e $8 \%$ não são influenciados por estes. Os participantes que não confiam, disseram que o fato de a cachaça possuir o selo de qualidade não garante que apresenta qualidade sensorial. Além disso, 20\% dos participantes não aprovam as cores e o design do selo da AMPAQ.

Quanto aos prêmios recebidos pela cachaça, $83 \%$ dos participantes consideram as premiações importantes, sendo um diferencial. Entretanto, 17\% têm opinião diferente e disseram que as premiações, muitas vezes, são antigas e têm pouca credibilidade.

\section{Conclusões}

Verificou-se que os participantes não aprovaram a garrafa de vidro de cor âmbar com tampa metálica tipo coroa (igual a da garrafa de cerveja) para acondicionar cachaça. Há preferência por garrafa de vidro transparente com capacidade igual a $700 \mathrm{~mL}$ e com tampa metálica rosqueável e por ilustrações do rótulo que estejam relacionadas com o processo produtivo de cachaça. A marca, selos de qualidade, premiações recebidas e as informações a respeito do local onde a cachaça foi produzida, do tempo de envelhecimento e do tipo de madeira do tonel no qual a cachaça foi envelhecida influenciam também na compra de cachaça. Observou-se ainda que os participantes apresentaram um padrão de preferência, sendo a maioria influenciada pelos mesmos fatores, no momento da compra de cachaça.

As informações obtidas neste estudo auxiliarão os produtores do setor de cachaça no desenvolvimento e/ou modificação de 
embalagens e rótulos, na escolha do tipo de madeira do tonel utilizado para envelhecer a cachaça, na determinação do tempo de envelhecimento e na elaboração de estratégias de marketing eficientes, contribuindo para aumentar a competitividade deste produto no mercado.

\section{Agradecimento}

Ao CNPq pela concessão de bolsa de pesquisadora à segunda autora.

\section{Referências bibliográficas}

AGUIAR, P. M. Aspectos físico-químicos de cachaças armazenadas em tonéis de carvalho. 73 f. Dissertação (Mestrado em Agroquímica e Agrobioquímica) - Universidade Federal de Lavras, Lavras, 2004.

ASSOCIAÇÃO BRASILEIRA DE BEBIDAS - ABRABE. 2009. Disponível em: <http://www.abrabe.org.br>. Acesso em: Ago. 2009.

ASSOCIAÇÃO MINEIRA DOS PRODUTORES DE CACHAÇA DE QUALIDADE - AMPAQ. 2009. Disponível em: <http://www. ampaq.com.br>. Acesso em: Ago. 2009.

BERNABÉU, R.; TENDERO, A. Preference structure for lamb meat consumers. A Spanish case study. Meat Science, v. 71, p. 464-470, 2005.

CARNEIRO, J. D. S. Impacto da embalagem de óleo de soja na intenção de compra do consumidor, via "conjoint analysis". 80 f. Dissertação (Mestrado em Ciência e Tecnologia de Alimentos) Universidade Federal de Viçosa, Viçosa, 2002.

COSTA, M. C. Tecnologias não convencionais e o impacto no comportamento do consumidor. 119 f. Dissertação (Mestrado em Ciência e Tecnologia de Alimentos)-Universidade Federal Rural do Rio de Janeiro, Rio de Janeiro, 1999.
DANTAS, M. I. S. Impacto da embalagem de couve (Brassica oleraceal cv. acephala) minimamente processada na intenção de compra do consumidor. 77 f. Dissertação (Mestrado em Ciência e Tecnologia de Alimtentos)-Universidade Federal de Viçosa, Viçosa, 2001.

DELIZA, R. The effects of expectation on sensory perception and acceptance. $198 \mathrm{f}$. Dissertação (PhD Thesis)-University of Reading, Inglaterra, 1996.

DELLA LUCIA, S. M. Conjoint analysis no estudo de mercado de café orgânico. 86 f. Dissertação (Mestrado em Ciência e Tecnologia de Alimentos) - Universidade Federal de Viçosa, Viçosa, 2005.

DELLA LUCIA, S. M.; MINIM, V. P. R. Grupo de foco. In: MINIM, V. P. R. (Ed.). Análise sensorial: estudos com consumidores. Viçosa: Editora UFV, 2006. cap. 4, p. 85-109.

FEDERAÇÃO NACIONAL DAS ASSOCIAÇÕES DOS PRODUTORES DE CACHAÇA DE ALAMBIQUE - FENACA. 2007. Disponível em: <http://www.fenaca.org.br>. Acesso em: jan. 2007.

MOSKOWITZ, H. et al. Sensory benefits, emotions and usage patterns for olives: using internet-based conjoint analysis and segmentation to understand patterns of response. Food Quality and Preference, v. 16, p. 369-382, 2004.

MURPHY, M.; COWAN, C.; HENCHION, M. Irish consumer preferences for honey: a conjoint approach. British Food Journal, v. 102, n. 8, p. 585-597, 2000.

REIS, R. C. Iogurte "light" sabor morango: equivalência de doçura, caracterização sensorial e impacto da embalagem na intenção de compra do consumidor. 143 f. Dissertação (Doutorado em Ciências e Tecnologia de Alimentos)-Universidade Federal de Viçosa, Viçosa, 2007.

SERVIÇO BRASILEIRO DE APOIO ÀS MICRO E PEQUENAS EMPRESAS - SEBRAE-MG. Diagnóstico da cachaça de Minas Gerais. Belo Horizonte, 2001. 259 p.

STEENKAMP, J. B. E. M. Conjoint measurement in ham quality evalution. Journal Agricultural Economies, v. 38, n. 3, p. 473-480, 1987. 